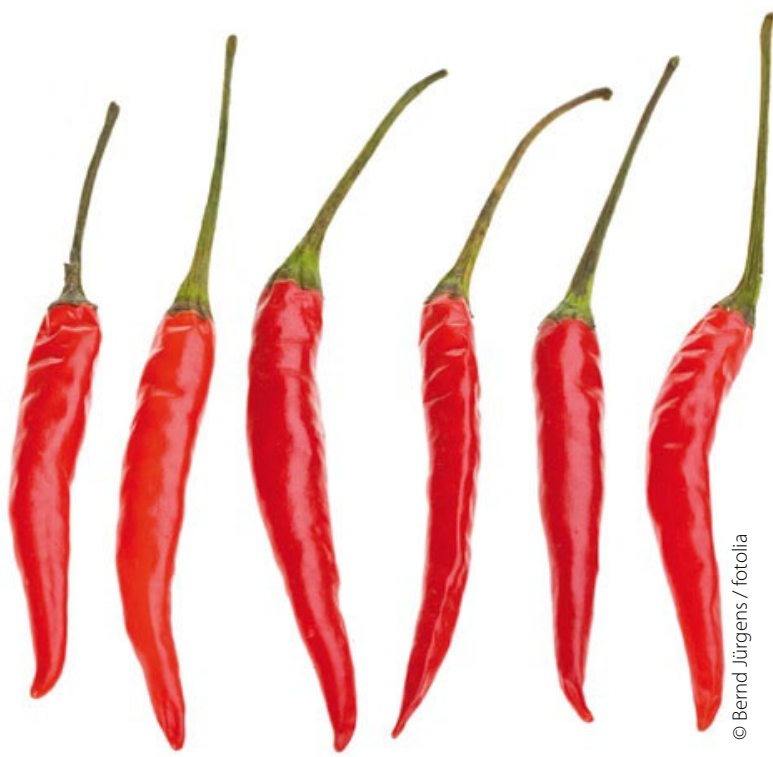

Mehrere Studien haben gezeigt, dass die Aufnahme von Nahrungsmitteln, die mit Cayennepfeffer gewürzt sind, mit einer negativen Energiebilanz einhergeht. Allerdings waren die Pfefferdosen, die in den Studien eingesetzt wurden, unrealistisch hoch (10 g!). Jetzt wurde untersucht, ob Cayennepfeffer auch in verträglicheren Mengen einen Einfluss auf das Körpergewicht haben könnte.

\title{
Cayennepfeffer als Appetitzügler
}

- Wissenschaftler der Purdue University in West Lafayette (USA) untersuchten 25 Männer und Frauen (Alter: 23,0 \pm 0,5 Jahre; BMI 22,6 $\left.\pm 0,3 \mathrm{~kg} / \mathrm{m}^{2}\right) .13$ bevorzugten auch im Alltag scharf gewürzte Speisen $(1,8 \pm 0,3 \mathrm{~g}$ Cayennepfeffer pro Mahlzeit), zwölf mochten es eher weniger feurig $(0,3 \pm 0,1 \mathrm{~g}$ Cayennepfeffer pro Mahlzeit). Die Probanden erhielten Testmahlzeiten, die entweder mit $1 \mathrm{~g}$ Cayennepfeffer gewürzt waren oder keinen Pfeffer enthielten. Über einen Temperatursensor, der am Nacken der Probanden befestigt wurde und über eine Kapsel, die sie zwölf Stunden vor der Testmahlzeit geschluckt hatten, wurden Haut- und Kerntemperatur registriert. Weiterhin wurden der Energieverbrauch in Ruhe nach der Mahlzeit sowie ein respiratorischer Quotient bestimmt.

Nach der cayennepfefferhaltigen Mahlzeit waren der Energieverbrauch und die Kerntemperatur erhöht sowie die Hauttemperatur und der respiratorische Quotient im Vergleich zur unge- würzten Mahlzeit erniedrigt. Bei Probanden, die keine gewohnheitsmäßigen Konsumenten von scharfen Speisen waren, waren Nahrungsaufnahme und Appetit nach der Testmahlzeit reduziert.

\section{Kommentar}

Wunder sind nach Meinung der Autoren allein von scharf gewürzten Speisen nicht zu erwarten. Zusammen mit anderen Maßnahmen - etwa Sport - kann scharfes Würzen aber möglicherweise ein zusätzlicher Tipp für diejenigen sein, die Übergewicht los werden oder gar nicht erst übergewichtig werden wollen.

K. MALBERG =

\section{Grundsätzlich H.-pylori-Eradikation bei Parkinsonpatienten?}

\section{Dass Helicobacter pylori nicht nur bei der Pathogenese des Ulkusleidens und des Magenkarzinoms, sondern auch bei einer Reihe anderer Erkrankungen wie Eisenmangelanämie oder chronischer Urtikaria eine wesentliche Rolle spielt, wird seit vielen Jahren diskutiert. Nach einer neueren Untersuchung profitie- ren auch Patienten mit einem idiopathischen Morbus Parkinson von der H.-pylori-Eradikation.}

— Untersucht wurde der Effekt der Helicobacter-pylori-Eradikationstherapie bei 30 Patienten mit einem idiopathischen Morbus Parkinson. Patienten, bei denen die Eradikation nicht erfolgreich war, zeigten im weiteren Verlauf eine deutliche Verschlechterung der neurologischen Symptomatik im Vergleich zu denjenigen Patienten, bei denen der Helicobacter pylori erfolgreich eradiziert werden konnte. Der positive Effekt war unabhängig von der Parkin- sonmedikation. Objektiviert wurde der günstige Effekt anhand der Schrittlänge und der Rigidität. Auch der weitere Verlauf in den darauffolgenden Jahren war nach einer erfolgreichen Eradikationstherapie günstiger.

\section{Kommentar}

Nur ein toter Helicobacter ist ein guter Helicobacter! Dieses Bonmot erfährt durch diese Studie eine neue Bestätigung. Doch bevor keine weiteren Studienergebnisse vorliegen, dürfte es wohl zum jetzigen Zeitpunkt noch nicht gerechtfertigt sein, bei allen Patienten mit einem idiopathischen Morbus Parkinson nach einer Helicobacter-pylori-Infektion zu fahnden und bei positivem Befund diese Patienten einer Eradikation zu unterziehen, zumal eine antibiotische Therapie auch mit einer Reihe von Risiken, insbesondere einer Clostridium-difficile-Infektion assoziiert ist.

P. STIEFELHAGEN .

\footnotetext{
- S.M. Dobbs et al.

Differential effect of Helicobacter pylori eradication on timetrends in brady/hypokinesia and rigidity in idiopathic parkinsonism. Report on completion of a randomised, double-blind, placebo-controlled efficacy study. Helicobacter 515 (2010) 279-294
} 